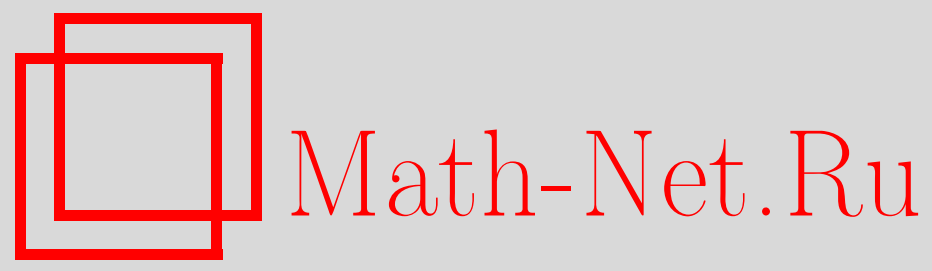

Е. Н. Яковлева, О бесконечных группах с точками, Дискрет. матем., 2002, том 14, выпуск 4, 153-157

DOI: https://doi.org/10.4213/dm269

Использование Общероссийского математического портала Math-Net.Ru подразумевает, что вы прочитали и согласны с пользовательским соглашением http://www.mathnet.ru/rus/agreement

Параметры загрузки:

IP: 3.81 .55 .215

26 апреля 2023 г., 15:02:50

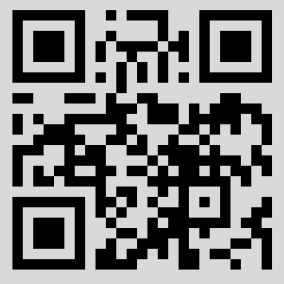


УДК 519.45

\title{
О бесконечных группах \\ с точками
}

\author{
(ㄷ) 2002 г. . Е. Н. Яковлева
}

\begin{abstract}
Рассматривается понятие точки группы, приводятся примеры групп с точками и доказывается теорема о расположении точек в подгруппах бесконечных групп.

Работа выполнена при поддержке Российского фонда фундаментальных исследований, проекты 99-01-00432 и 02-01-00078, и гранта №9 Шестого конкурса научных проектов молодых ученых РАН 1999 r.
\end{abstract}

Точками группы $G$ называются элементы конечного порядка следующего типа:

- единица группы есть точка в том и только в том случае, когда множество элементов конечного порядка из $G$ конечно;

- неединичный элемент $a$ есть точка, если для всякой неединичной конечной подгруппы $K<G$, нормализуемой элементом $a$, множество конечных подгрупп из $N_{G}(K)$, содержащих $a$, конечно.

Точка $a$ называется тривиальной, если множество конечных подгрупп из $G$, содержащих $a$, конечно.

Определение точки введено В. П. Шунковым в [1].

Группы без инволюций, обладающие точками, рассматривались в [10]. Некоторые свойства групп с инволющиями, содержащих точки, описаны в [11].

Приведем примеры групп, обладающих точками.

- В конечных группах каждый элемент является точкой.

- Группа Новикова-Адяна, периодические произведения Адяна конечных групп без инволюций, периодический монстр Ольшанского являются примерами групा, в которых каждый неединичный элемент является точкой.

- Единичная группа и группа без кручений - группы с единственной точкой.

- Группа с конечной периодической частью - группа, в которой каждый элемент конечного порядка является точкой.

- Свободное произведение неединичной конечной группы и любой другой неединичной группы - группа с бесконечным множеством точек. 
- Пусть $T_{1}, T_{2}, \ldots$ - бесконечная последовательность конечных фробениусовых групा с одним и тем же неинвариантным множителем $H$, то есть,

$$
T_{n}=F_{n} \lambda H, \quad n=1,2, \ldots
$$

Свободное произведение $G$ групп этой последовательности с объединенной подгруппой $H$ является группой с нетривиальной точкой.

Существуют группы, не обладающие точками.

Теорема 1. Никакая группа не содержит бесконечной локально конечной подгруппы с точками.

Доказательство. Рассмотрим группу $G$ и в ней бесконечную локально конечную подгруппу $H$. Возможны два случая:

(1) $H$ - группа без инволюций, и тогда по теореме Файта-Томпсона все ее конечные подгруппы разрешимы,

(2) $H$ - группа с инволюциями.

Пусть сначала $H$ - локально конечная группа с разрешимыми конечными подгруппами и $a-$ некоторый элемент группы $H$. Предположим, что $a$ является точкой группы $H$. Тогда централизатор $C_{H}(a)$ конечен в силу периодичности группы $\boldsymbol{H}$. Для любого неединичного элемента $b \in C_{H}(a)$ простого порядка централизатор $C_{H}(b)$ конечен. Действительно, предположим, что $C_{H}(b)$ бесконечен и $a \in C_{H}(b)$. Очевидно, что $a$ является точкой группы $C_{H}(b)$. В $C_{H}(b)$ есть нормальная конечная подгруппа $(b)$. Так как централизатор $C_{H}(b)$ локально конечен, в $C_{H}(b)$ найдется бесконечное множество конечных подгрупп, содержащих элемент $a$. Это невозможно.

Действительно, покажем, что никакая группа $U$ не может одновременно содержать бесконечное множество конечных подгрупп с нетривиальным пересечением, содержащим точку $z$, и нетривиальную конечную нормальную подгруппу. Так как в группе $U$ имеется бесконечное число элементов конечного порядка, то $z \neq e$ по определению точки. Пусть группа $U$ содержит бесконечное множество конечных подгрупп с нетривиальным пересечением, содержащим точку $z$ и нетривиальную конечную нормальную подгруппу $D$. Так как $D$ является нормальной подгруппой группы $U$, то

$$
N_{U}(D)=U
$$

и множество конечных подгрупп в $N_{U}(D)$, содержащих $z$, бесконечно, то есть $z$ не является точкой группы $U$. 
Рассмотрим множество

$$
\begin{aligned}
\pi & =\pi(a) \\
& =\left\{p_{1}, p_{2}, \ldots, p_{n}\right\}
\end{aligned}
$$

и множество

$$
\left\{h_{1}, h_{2}, \ldots, h_{n}\right\}
$$

где

$$
h_{i} \in(a), \quad\left|h_{i}\right|=p_{i}, \quad i=1,2, \ldots, n
$$

Так как $h_{i} \in C_{H}(a)$, то, как показано выше,

$$
\left|C_{H}\left(h_{i}\right)\right|<\infty, \quad i=1,2, \ldots, n .
$$

Так как $H$ - периодическая локально разрешимая группа и $h_{i}$ - почти регулярный элемент простого порядка, все силовские $p_{i}$-подгруппы из $H$ являются черниковскими, $i=1,2, \ldots, n[3]$ и $H / O_{p_{i}^{\prime}}(H)$ - черниковские группы, $i=1,2, \ldots, n$ [5]. Поэтому по теореме Ремака (см. [4]) и в силу свойств черниковских групा факторгруппа $H / Z$, где

$$
Z=\bigcap_{i=1}^{n} o_{p_{i}^{\prime}}(H),
$$

также является черниковской.

Если бы подгруппа $Z$ была черниковской, то группа $H$, являясь расширением черниковской группы с помощью черниковской группы, была бы черниковской группой, которая не может обладать точками. Действительно, в силу свойств черниковских групा в бесконечной черниковской группе каждый элемент содержится в бесконечном множестве конечных подгрупп. Так как в любой бесконечной черниковской группе найдется конечная нормальная подгруппа, по доказанному выше, бесконечная черниковская группа не содержит точек. Но $a$ - точка группы $H$, следовательно, $Z$ не является черниковской группой.

Рассмотрим группу $Z \lambda(a)$. Так как $C_{Z}\left(h_{1}\right) \lambda(a)$ - конечная разрешимая группа и $C_{Z}\left(h_{1}\right)-\pi^{\prime}$-подгруппа, для любого $q \in \pi\left(C_{Z}\left(h_{1}\right)\right)$ найдется силовская $q$-подгруппа $Q$ из $C_{Z}\left(h_{1}\right)$, нормализуемая элементом $a$. Подгруппа $Q$ содержится в некоторой силовской $q$-подгруппе $L$ из $Z$. Если $L$ не является черниковской группой, то по теореме Блекберна (см. [9]), согласно которой в локально конечной $p$-группе $G$, если централизатор некоторой конечной подгруппы черниковский, то и сама группа черниковская, получаем, что нормализатор $N_{L}(Q)$ не является черниковской группой. Но $N_{L}(Q)$ локально конечен и содержит точку $a$. Получили противоречие, так как никакая группа не может содержать одновременно бесконечное множество конечных подгрупп с нетривиальным пересечением, содержащим точку $a$, и нетривиальную конечную нормальную подгруппу.

Значит, $L$ - черниковская группа. Если в качестве $L$ взять проекционную силовскую $q$-подгруппу, то все силовские подгруппы из $Z$ по простым делителям порядка группы 
$C_{Z}\left(h_{1}\right)$ являются черниковскими [2]. Еще раз применяя теорему Ремака, получаем, что $Z / Z_{1}$ - черниковская группа, где

$$
Z_{1}=\bigcap_{q \in \pi\left(C_{Z}\left(h_{1}\right)\right)} O_{q}^{\prime}(Z) .
$$

Группа $Z_{1}$ бесконечна, так как в противном случае $Z$ была бы черниковской. Рассмотрим групту $Z_{1} \lambda\left(h_{1}\right)$. Очевидно, что элемент $h_{1}$ действует регулярно на $Z_{1}$. Так как $Z_{1}$ - локально конечная группа, она является нильпотентной. Действительно, пусть $K$ произвольная конечная подгруппа из $Z_{1}$. В силу локальной конечности $Z_{1}$, подгруппу $K$ можно включить в конечную подгруппу

$$
M=\operatorname{gr}\left(K, K^{h_{1}}, \ldots, K_{1}^{h_{1}^{p_{1}-1}}\right)
$$

для которой действие элементом $h_{1}$ является регулярным автоморфизмом простого порядка $p_{1}$. По теореме Хигмана-Томпсона $[6,7]$ всякая конечная группа, обладающая регулярным автоморфизмом простого порядка, нильпотентна и длина ее верхнего центрального ряда ограничена числом, зависящим только от $p$. Следовательно, гругпа $M$ нильпотентна и длина ее верхнего центрального ряда ограничена числом $s$, зависящим только от $p_{1}$. Это означает, что для любых элементов

$$
a_{1}, a_{2}, \ldots, a_{s} \in M
$$

коммутатор

$$
\left[a_{1}, a_{2}, \ldots, a_{s}\right]=1
$$

Так как $s$ не зависит от выбора подгруппы $K$, группа $Z_{1}$ нильпотентна. Рассмотрим группу

$$
R=Z_{1} \lambda(a)
$$

Группа $Z_{1}$ является бесконечной периодической нильпотентной группой, следовательно, она обладает нетривиальной нормальной подгруппой $S$ такой, что $N_{R}(S)$ бесконечен и содержит точку $a$. Учитывая, что $N_{R}(S)$ - локально конечная группа, получаем противоречие. В этом случае теорема доказана.

Пусть теперь $H$ содержит инволюции и $a$ - точка группы $H$. Нормализатор точки обладает конечной периодической частью, поэтому $C_{H}(a)$ конечен. По следствию А1 теоремы Хартли в [8] локально конечная группа, содержащая элемент с конечным централизатором, обладает локально разрешимой нормальной подгруппой конечного индекса. Тогда группа $H$ обладает локально разрешимой нормальной подгруппой $M$ конечного индекса. По доказанному выше, подгруппа $M$ не содержит точек.

Рассмотрим группу $M \cdot(a)$. Очевидно, что эта группа локально разрешима и $a$ яІвляется в ней точкой. Но, по вышесказанному, группа $M \cdot(a)$ не обладает точками. Полученное противоречие означает, что группа $H$ не содержит точек. Теорема доказана. 


\section{Список литературы}

1. Шунков В. П., Мр-группы. Наука, Москва, 1990.

2. Каргаполов М. И., Мерзляков Ю. И., Основы теории групп. Наука, Москва, 1982.

3. Шунков В. П., О локально конечной группе с экстремальными силовскими р-подгруппами по некоторому простому числу р. Сибирский матем. журнал (1967) 8, №1, 213-229.

4. Шунков В. П., Об одном классе р-групп. Алгебра и логика (1970) 9, №4, 484-496.

5. Черников С. Н., Условия конечности в общей теории групп. Успехи матем. наук (1959) 14, №5, 45-96.

6. Higman G., Groups and rings having automorphisms without nontrivial fixed points. J. London Math. Soc. (1957), №32, 321-334.

7. Thompson J. G., Finite groups with fixed point free automorphisms of prime order. Proc. Nat. Amer. Sci. USA (1959), №45, 578-581.

8. Hartley B. A., A general Brauer-Fowler theorem and centralizers in locally finite groups Pacific $J$. Math. (1992), №152, 101-117.

9. Blackburn N., Some remarks on Chernikov p-groups. III. J. Math. (1962), №6, 421-433.

10. Сенашов В. И., Шунков В. П., О группах с конечной периодической частью. Алгебра и логика (1983) 22, №1, 93-112.

11. Шунков В. П., Группы с инволюциями. Препринт ВЦ СО АН СССР, Красноярск, 1986.

Статья поступила 27.09.2001. 